\title{
OPTIMALITY CONDITIONS FOR SEMILINEAR PARABOLIC EQUATIONS WITH CONTROLS IN LEADING TERM*
}

\author{
HONGWEI LOU ${ }^{1}$
}

\begin{abstract}
An optimal control problem for semilinear parabolic partial differential equations is considered. The control variable appears in the leading term of the equation. Necessary conditions for optimal controls are established by the method of homogenizing spike variation. Results for problems with state constraints are also stated.
\end{abstract}

Mathematics Subject Classification. 49K20, 35B27.

Received August 7, 2009. Revised February 18, 2010.

Published online August 23, 2010.

\section{INTRODUCTION}

We will give necessary conditions of optimal controls for parabolic partial differential equation (PDE, for short) with leading term containing controls. This is an analogue of the result we got for elliptic PDE with controls in the leading term [11]. Let us consider the following controlled parabolic PDE of divergence form:

$$
\left\{\begin{array}{l}
\partial_{t} z(t, x)-\nabla \cdot(A(t, x, u(t, x)) \nabla z(t, x))=f(t, x, z(t, x), u(t, x)), \quad \text { in } \Omega_{T}, \\
z(t, x)=0, \quad \text { on }[0, T] \times \partial \Omega \\
z(0, x)=z_{0}(x), \quad \text { in } \Omega,
\end{array}\right.
$$

where $\Omega_{T}=(0, T) \times \Omega, T>0$ and $\Omega \subseteq \mathbb{R}^{n}$ is a bounded domain with a smooth boundary $\partial \Omega, A: \Omega_{T} \times U \rightarrow \mathbb{R}^{n \times n}$ is a map taking values in the set of all positive definite matrices, $f: \Omega_{T} \times \mathbb{R} \times U \rightarrow \mathbb{R}$, with $U$ being a separable metric space and $z_{0}(\cdot) \in L^{\infty}(\Omega)$. Function $u(\cdot)$, called a control, is taken from the set

$$
\mathcal{U} \equiv\left\{w: \Omega_{T} \rightarrow U \mid w(\cdot) \text { is measurable }\right\}
$$

Under some mild conditions, corresponding to a $u(\cdot) \in \mathcal{U},(1.1)$ admits a unique weak solution $z(\cdot) \equiv z(\cdot ; u(\cdot))$ which is called the state. We measure the performance of the control by the following cost functional

$$
J(u(\cdot))=\int_{\Omega_{T}} f^{0}(t, x, z(t, x), u(t, x)) \mathrm{d} t \mathrm{~d} x
$$

for some given map $f^{0}: \Omega_{T} \times \mathbb{R} \times U \rightarrow \mathbb{R}$. Our optimal control problem is stated as follows.

\footnotetext{
Keywords and phrases. Optimal control, necessary conditions, parabolic equation, homogenized spike variation.

* The author was supported in part by NSFC (No. 10671040 and 10831007), and FANEDD (No. 200522).

${ }^{1}$ School of Mathematical Sciences, and LMNS, Fudan University, Shanghai 200433, P.R. China. hwlou@fudan.edu.cn
} 
Problem (C). Find a $\bar{u}(\cdot) \in \mathcal{U}$ such that

$$
J(\bar{u}(\cdot))=\inf _{u(\cdot) \in \mathcal{U}} J(u(\cdot))
$$

Any $\bar{u}(\cdot) \in \mathcal{U}$ satisfying (1.3) is called an optimal control, and the corresponding $\bar{z}(\cdot) \equiv z(\cdot ; \bar{u}(\cdot))$ is called an optimal state. The pair $(\bar{z}(\cdot), \bar{u}(\cdot))$ is called an optimal pair. When $A(t, x, u) \equiv A(t, x)$, Problem (C) has been studied by many authors, see [10] and the references cited therein. Works concerning the elliptic cases with leading term containing controls can be founded in [4-7,11-14], etc. However, it seems that there are only few works devoted to parabolic cases (see [3,15], etc.).

In this paper, we make the following assumptions.

(S1) Let $T>0$ and $\Omega$ be a bounded domain in $\mathbb{R}^{n}$ with a smooth boundary $\partial \Omega$.

(S2) Let $U$ be a separable metric space.

(S3) Functions $A(t, x, v)=\left(a_{i j}(t, x, v)\right)$ take values in the set $\mathcal{S}_{+}^{n}$ of $n \times n$ (symmetric) positive definite matrices, which are measurable in $(t, x) \in \Omega_{T}$ and continuous in $v \in U$. Moreover, there exist $\Lambda \geq \lambda>0$ such that for almost all $(t, x) \in \Omega_{T}$,

$$
\lambda|\xi|^{2} \leq\langle A(t, x, v) \xi, \xi\rangle \leq \Lambda|\xi|^{2}, \quad \forall \xi \in \mathbb{R}^{n}, v \in U,
$$

where $\langle\cdot, \cdot\rangle$ stands for the inner product in $\mathbb{R}^{n}$.

(S4) Functions $f(t, x, z, v)$ and $f_{z}(t, x, z, v)$ are measurable in $(t, x)$, and continuous in $(z, v) \in \mathbb{R} \times U$. Moreover, there exits a constant $M>0$ such that

$$
z f(t, x, z, v) \leq M\left(z^{2}+1\right), \quad \forall(t, x, z, v) \in \Omega_{T} \times \mathbb{R} \times U
$$

and for any $R>0$, there exists an $M_{R}>0$ such that

$$
|f(t, x, z, v)|+\left|f_{z}(t, x, z, v)\right| \leq M_{R}, \quad \text { a.e. }(t, x, v) \in \Omega_{T} \times U,|z| \leq R .
$$

(S5) Functions $f^{0}(t, x, z, v)$ and $f_{z}^{0}(t, x, z, v)$ are measurable in $(t, x)$, and continuous in $(z, v) \in \mathbb{R} \times U$. Moreover, for any $R>0$, there exists a $K_{R}>0$ such that

$$
\left|f^{0}(t, x, z, v)\right|+\left|f_{z}^{0}(t, x, z, u)\right| \leq K_{R}, \quad \text { a.e. }(t, x, v) \in \Omega_{T} \times U,|z| \leq R .
$$

Our main result is the following.

Theorem 1.1. Let (S1)-(S5) hold and $z_{0} \in L^{\infty}(\Omega)$. Let $(\bar{z}(\cdot), \bar{u}(\cdot))$ be an optimal pair of Problem $(\mathrm{C})$. Let $\bar{\psi}(\cdot)$ be the weak solution of the following adjoint equation

$$
\left\{\begin{aligned}
\partial_{t} \bar{\psi}(t, x)+\nabla \cdot(A(t, x, \bar{u}(t, x)) \nabla \bar{\psi}(t, x))=f_{z}^{0}(t, x, \bar{z}(t, x), \bar{u}(t, x)) & \\
& -f_{z}(t, x, \bar{z}(t, x), \bar{u}(t, x)) \bar{\psi}(t, x), \quad \text { in } \Omega_{T}, \\
\bar{\psi}(t, x)=0, \quad \text { on }[0, T] \times \partial \Omega, & \text { in } \Omega .
\end{aligned}\right.
$$

Then

$$
\begin{gathered}
H(t, x, \bar{z}(t, x), \bar{\psi}(t, x), \nabla \bar{z}(t, x), \nabla \bar{\psi}(t, x), \bar{u}(t, x))-H(t, x, \bar{z}(t, x), \bar{\psi}(t, x), \nabla \bar{z}(t, x), \nabla \bar{\psi}(t, x), v) \\
\geq \frac{1}{2}\left|A(t, x, v)^{-\frac{1}{2}}(A(t, x, \bar{u}(t, x))-A(t, x, v)) \nabla \bar{z}(t, x)\right|\left|A(t, x, v)^{-\frac{1}{2}}(A(t, x, \bar{u}(t, x))-A(t, x, v)) \nabla \bar{\psi}(t, x)\right| \\
+\frac{1}{2}\left\langle A(t, x, v)^{-\frac{1}{2}}(A(t, x, \bar{u}(t, x))-A(t, x, v)) \nabla \bar{z}(t, x), A(t, x, v)^{-\frac{1}{2}}(A(t, x, \bar{u}(t, x))-A(t, x, v)) \nabla \bar{\psi}(t, x)\right\rangle, \\
\forall v \in U, \quad \text { a.e. }(t, x) \in \Omega_{T},
\end{gathered}
$$


where

$$
\begin{gathered}
H(t, x, z, \psi, \xi, \eta, v)=\langle\psi, f(t, x, z, v)\rangle-f^{0}(t, x, z, v)-\langle A(t, x, v) \xi, \eta\rangle \\
(t, x, z, \psi, \xi, \eta, v) \in[0, T] \times \Omega \times \mathbb{R} \times \mathbb{R} \times \mathbb{R}^{n} \times \mathbb{R}^{n} \times U .
\end{gathered}
$$

Since the right hand side of (1.9) is always nonnegative, (1.9) implies

$$
\begin{array}{r}
H(t, x, \bar{z}(t, x), \bar{\psi}(t, x), \nabla \bar{z}(t, x), \nabla \bar{\psi}(t, x), \bar{u}(t, x))=\max _{v \in U} H(t, x, \bar{z}(t, x), \bar{\psi}(t, x), \nabla \bar{z}(t, x), \nabla \bar{\psi}(t, x), v), \\
\forall v \in U, \quad \text { a.e. }(t, x) \in \Omega_{T} .
\end{array}
$$

When $A(t, x, v) \equiv A(t, x)$, the right hand side of (1.9) is zero, thus, the result automatically recovers those for the classical semilinear case without state constraints [10].

Since $U$ is not necessarily convex, it is well-known that people usually use spike variations to derive necessary conditions for optimal controls. Such a spike variation technique does not directly work for problems with leading term containing the control. To overcome the difficulty, we adopt the idea of homogenization for PDEs to carefully select some special type spike variations of controls so that we can have desired "differentiability" of the state with respect to the control. We can see in [11] that such a method is useful for the cases of elliptic PDEs. The main idea to treat parabolic case is same to that for elliptic case. However, there are some new difficulties in studying properties of variational equations.

Comparing Theorem 1.1 and the corresponding result for elliptic case in [11], we can see that they are similar when $n \geq 2$ and slightly different when $n=1$. More precisely, Theorem 1.1 of this paper is very similar to Theorem 1.1 in [11] for high dimensional cases. In particular, for parabolic case with $n=1$, instead of

$$
\begin{aligned}
& H\left(t, x, \bar{z}(t, x), \bar{\psi}(t, x), \bar{z}_{x}(t, x), \bar{\psi}_{x}(t, x), \bar{u}(t, x)\right)-H\left(t, x, \bar{z}(t, x), \bar{\psi}(t, x), \bar{z}_{x}(t, x), \bar{\psi}_{x}(t, x), v\right) \\
\geq & \frac{(A(t, x, \bar{u}(t, x))-A(t, x, v))^{2}}{A(t, x, v)} \bar{z}_{x}(t, x) \bar{\psi}_{x}(t, x), \quad \forall v \in U, \quad \text { a.e. }(t, x) \in \Omega_{T},
\end{aligned}
$$

we have (1.9), i.e.,

$$
\begin{aligned}
& H\left(t, x, \bar{z}(t, x), \bar{\psi}(t, x), \bar{z}_{x}(t, x), \bar{\psi}_{x}(t, x), \bar{u}(t, x)\right)-H\left(t, x, \bar{z}(t, x), \bar{\psi}(t, x), \bar{z}_{x}(t, x), \bar{\psi}_{x}(t, x), v\right) \\
\geq & \frac{(A(t, x, \bar{u}(t, x))-A(t, x, v))^{2}}{A(t, x, v)}\left[\bar{z}_{x}(t, x) \bar{\psi}_{x}(t, x)\right]^{+}, \quad \forall v \in U, \quad \text { a.e. }(t, x) \in \Omega_{T} .
\end{aligned}
$$

One can see that (1.12) is similar to the corresponding result for elliptic case with $n=1$, while (1.9) (i.e., (1.13)) is similar to the corresponding result for elliptic case with $n \geq 2$. We mention that for elliptic cases with $n \geq 2$, the corresponding right hand of (1.9) follows from a fact given in Lemma 2.5. While for parabolic case with $n=1$, the right hand of (1.9) (i.e., (1.13)) follows in a different way. In fact, it follows from (1.12) and

$$
\begin{array}{r}
H\left(t, x, \bar{z}(t, x), \bar{\psi}(t, x), \bar{z}_{x}(t, x), \bar{\psi}_{x}(t, x), \bar{u}(t, x)\right)-H\left(t, x, \bar{z}(t, x), \bar{\psi}(t, x), \bar{z}_{x}(t, x), \bar{\psi}_{x}(t, x), v\right) \geq 0, \\
\forall v \in U, \quad \text { a.e. }(t, x) \in \Omega_{T} .
\end{array}
$$

From the proof of Theorem 1.1, one can see that (1.12) can be yielded from using spike variation along spacedirection and (1.14) can be yielded from using spike variation along time-direction (see (3.24)).

Another difference between parabolic cases and elliptic cases appear in that there are three possible types of homogenized equations for parabolic cases when taking a different scale for the time and the space variables, while there is only one type of homogenized equations for elliptic cases. Difficulty occurs in analyzing the second type of homogenized equations (see the proof of Lem. 2.2 for details). Despite the different types of homogenized equations, the variational equations are same and we finally get same optimality conditions for 
the three cases. Nevertheless, we think results of this paper will be useful to analyze the second-order variational equations, which is a problem more difficult than that for first-order variational equations.

The rest of the paper is organized as follows. In Section 2, we present some preliminary results. Section 3 is devoted to a proof of our main result. Problem with state constraints will be discussed in Section 4.

\section{Preliminaries}

In this section, we will give some preliminary results needed in proving Theorem 1.1. For $Y=\left[0, \alpha_{1}\right] \times$ $\left[0, \alpha_{2}\right] \times \ldots\left[0, \alpha_{n}\right]$, a function $g(x)$ on $\mathbb{R}^{n}$ is called $Y$-periodic if it admits period $\alpha_{j}$ in the direction $x_{j}(j=$ $1,2, \ldots, n)$.

Lemma 2.1. Let $r>0, \delta \in(0,1)$ and $(\mathrm{S} 1)$ hold. Let $h(\cdot) \in L^{2}\left(\Omega_{T}\right)$, and $A^{m}(\cdot)=\left(a_{i j}^{m}(\cdot)\right) \in L^{\infty}\left(\Omega_{T} ; \mathcal{S}_{+}^{n}\right)$ such that for some $\Lambda \geq \lambda>0$,

$$
\lambda|\xi|^{2} \leq\left\langle A^{m}(t, x) \xi, \xi\right\rangle \leq \Lambda|\xi|^{2}, \quad \forall \xi \in \mathbb{R}^{n},(t, x) \in \Omega_{T}, m=1,2,3,4 .
$$

Define

$$
G(t, x, s, y) \equiv\left(g_{i j}(t, x, s, y)\right) \equiv\left(g_{i j}\left(t, x, s, y_{1}\right)\right)=\left\{\begin{array}{l}
A^{1}(t, x), \text { if }\left(\{s\},\left\{y_{1}\right\}\right) \in[\delta, 1) \times[\delta, 1), \\
A^{2}(t, x), \text { if }\left(\{s\},\left\{y_{1}\right\}\right) \in[0, \delta) \times[\delta, 1), \\
A^{3}(t, x), \text { if }\left(\{s\},\left\{y_{1}\right\}\right) \in[\delta, 1) \times[0, \delta), \\
A^{4}(t, x), \text { if }\left(\{s\},\left\{y_{1}\right\}\right) \in[0, \delta) \times[0, \delta),
\end{array}\right.
$$

where $\{a\}$ denotes the decimal part of a real number a. For $\varepsilon>0$, let $z^{\varepsilon}(\cdot) \in L^{2}\left(0, T ; H_{0}^{1}(\Omega)\right)$ be the weak solution of

$$
\left\{\begin{array}{l}
\partial_{t} z^{\varepsilon}(t, x)-\nabla \cdot\left[G\left(t, x, \frac{t}{\varepsilon^{r}}, \frac{x}{\varepsilon}\right) \nabla z^{\varepsilon}(t, x)\right]=h(t, x), \quad \text { in } \Omega_{T}, \\
z^{\varepsilon}(t, x)=0, \quad \text { on }[0, T] \times \partial \Omega, \\
z^{\varepsilon}(0, x)=z_{0}(x), \quad \text { in } \Omega,
\end{array}\right.
$$

with $z_{0}(\cdot) \in L^{\infty}(\Omega)$. Then

$$
z^{\varepsilon}(\cdot) \rightarrow z(\cdot), \quad \text { weakly in } \quad L^{2}\left(0, T ; H_{0}^{1}(\Omega)\right)
$$

with $z(\cdot)$ being the weak solution of

$$
\left\{\begin{array}{l}
\partial_{t} z(t, x)-\nabla \cdot(Q(t, x) \nabla z(t, x))=h(t, x), \quad \text { in } \Omega_{T} \\
z(t, x)=0, \quad \text { on }[0, T] \times \partial \Omega \\
z(0, x)=z_{0}(x), \quad \text { in } \Omega
\end{array}\right.
$$

and $Q(\cdot)=\left(q_{i j}(\cdot)\right) \in L^{\infty}\left(\Omega_{T} ; \mathcal{S}_{+}^{n}\right)$ being given by

$$
q_{i j}(t, x)=\int_{0}^{1} \mathrm{~d} y_{1} \int_{0}^{1}\left(g_{i j}\left(t, x, s, y_{1}\right)+g_{i 1}\left(t, x, s, y_{1}\right) \partial_{y_{1}} \varphi^{j}\left(t, x, s, y_{1}\right)\right) \mathrm{d} s, \quad 1 \leq i, j \leq n
$$

where $\varphi^{k}(t, x, \cdot) \in L_{\#}^{2}\left(0,1 ; W_{\#}^{1,2}(0,1) / \mathbb{R}\right)$, \# means the function is $[0,1]$ periodic.

For $r<2, \varphi^{k}(t, x, \cdot)$ is the unique solution of

$$
\partial_{y_{1}}\left(g_{1 k}\left(t, x, s, y_{1}\right)+g_{11}\left(t, x, s, y_{1}\right) \partial_{y_{1}} \varphi^{k}\left(t, x, s, y_{1}\right)\right)=0 \text {. }
$$

For $r=2, \varphi^{k}(t, x, \cdot)$ is the solution of

$$
\partial_{s} \varphi^{k}\left(t, x, s, y_{1}\right)-\partial_{y_{1}}\left(g_{1 k}\left(t, x, s, y_{1}\right)+g_{11}\left(t, x, s, y_{1}\right) \partial_{y_{1}} \varphi^{k}\left(t, x, s, y_{1}\right)\right)=0
$$


While for $r>2, \varphi^{k}(t, x, s, y)=\varphi^{k}\left(t, x, y_{1}\right)$ is the solution of

$$
\partial_{y_{1}}\left(\int_{0}^{1} g_{1 k}\left(t, x, s, y_{1}\right) \mathrm{d} s+\int_{0}^{1} g_{i j}\left(t, x, s, y_{1}\right) \mathrm{d} s \partial_{y_{1}} \varphi^{k}\left(t, x, y_{1}\right)\right)=0
$$

Proof. The above proposition is a corollary of Theorem 2.1 in [2], Chapter 2 (see also Rem. 1.1 and "Comments and Problems" there). The result can also be got by the technique of two scale convergence $[1,8]$. According to $[2]$,

$$
q_{i j}(t, x)=\int_{[0,1]^{n}} \mathrm{~d} y \int_{0}^{1}\left(g_{i j}(t, x, s, y)+\sum_{k=1}^{n} g_{i k}(t, x, s, y) \partial_{y_{k}} \varphi^{j}(t, x, s, y)\right) \mathrm{d} s, \quad 1 \leq i, j \leq n .
$$

For $r<2, \varphi^{k}(t, x, \cdot) \in L_{\#}^{2}\left(0,1 ; W_{\#}^{1,2}\left((0,1)^{n}\right) / \mathbb{R}\right)$ is the unique solution of

$$
\sum_{i, j=1}^{n} \partial_{y_{i}}\left(g_{i j}(t, x, s, y) \delta_{j k}+g_{i j}(t, x, s, y) \partial_{y_{j}} \varphi^{k}(t, x, s, y)\right)=0
$$

where $\delta_{i j}$ equals to 1 if $i=j$ and 0 if $i \neq j$.

For $r=2, \varphi^{k}(t, x, \cdot)$ is the solution of

$$
\partial_{s} \varphi^{k}(t, x, s, y)-\sum_{i, j=1}^{n} \partial_{y_{i}}\left(g_{i j}(t, x, s, y) \delta_{j k}+g_{i j}(t, x, s, y) \partial_{y_{j}} \varphi^{k}(t, x, s, y)\right)=0
$$

While for $r>2, \varphi^{k}(t, x, s, y)=\varphi(t, x, y)$ is the solution of

$$
\sum_{i, j=1}^{n} \partial_{y_{i}}\left(\int_{0}^{1} g_{i j}(t, x, s, y) \delta_{j k} \mathrm{~d} s+\int_{0}^{1} g_{i j}(t, x, s, y) \mathrm{d} s \partial_{y_{j}} \varphi^{k}(t, x, y)\right)=0
$$

Since $G(t, x, s, y)$ is independent of $y_{2}, y_{3}, \ldots, y_{n}$, we must have $\varphi^{k}(t, x, s, y)=\varphi^{k}\left(t, x, s, y_{1}\right)$ and consequently, $(2.10)-(2.13)$ becomes $(2.6)-(2.9)$.

The following lemma concerns the "derivative" of $q_{i j}$ in $\delta=0$.

Lemma 2.2. Let $r>0, \Lambda>\lambda>0$. Assume $\Lambda \geq a_{m} \geq \lambda,\left|b_{m}\right| \leq \Lambda,\left|c_{m}\right| \leq \Lambda(m=1,2,3,4)$. Let $\delta \in(0,1)$ and

$$
\left(a^{\delta}(s, y), b^{\delta}(s, y), c^{\delta}(s, y)\right)= \begin{cases}\left(a_{1}, b_{1}, c_{1}\right), & \text { if }(\{s\},\{y\}) \in[\delta, 1) \times[\delta, 1), \\ \left(a_{2}, b_{2}, c_{2}\right), & \text { if }(\{s\},\{y\}) \in[0, \delta) \times[\delta, 1), \\ \left(a_{3}, b_{3}, c_{3}\right), & \text { if }(\{s\},\{y\}) \in[\delta, 1) \times[0, \delta), \\ \left(a_{4}, b_{4}, c_{4}\right), & \text { if }(\{s\},\{y\}) \in[0, \delta) \times[0, \delta) .\end{cases}
$$




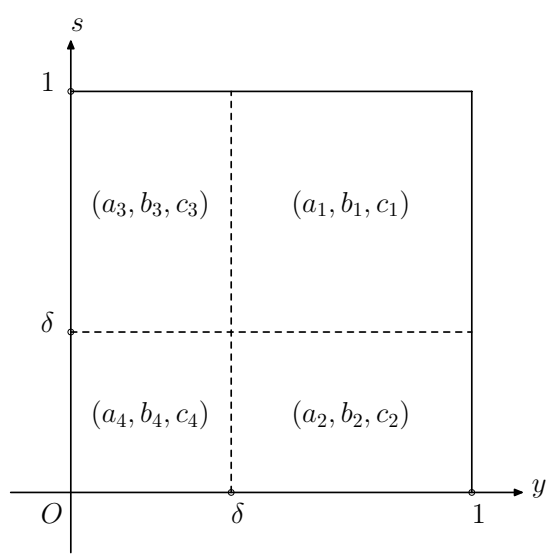

Let $\phi_{1}^{\delta}(\cdot) \in L_{\#}^{2}\left(0,1 ; W_{\#}^{1,2}(0,1) / \mathbb{R}\right)$ be the solution of

$$
\partial_{y}\left(b^{\delta}(s, y)+a^{\delta}(s, y) \partial_{y} \phi_{1}^{\delta}(s, y)\right)=0
$$

$\phi_{2}^{\delta}(\cdot)$ be the solution of

$$
\partial_{s} \phi_{2}^{\delta}(s, y)-\partial_{y}\left(b^{\delta}(s, y)+a^{\delta}(s, y) \partial_{y} \phi_{2}^{\delta}(s, y)\right)=0
$$

and $\phi_{3}^{\delta}(s, y) \equiv \phi_{3}^{\delta}(y)$ be the solution of

$$
\partial_{y}\left(\int_{0}^{1} b^{\delta}(s, y) \mathrm{d} s+\int_{0}^{1} a^{\delta}(s, y) \mathrm{d} s \partial_{y} \phi_{3}^{\delta}(y)\right)=0 .
$$

Then there exists a constant $C=C(\Lambda, \lambda)$, independent of $\delta \in(0,1)$, such that

$$
\left|\frac{1}{\delta} \int_{0}^{1} \mathrm{~d} y \int_{0}^{1} c^{\delta}(s, y) \partial_{y} \phi_{k}^{\delta}(s, y) \mathrm{d} s+\frac{\left(b_{3}-b_{1}\right)\left(c_{3}-c_{1}\right)}{a_{3}}\right| \leq C \sqrt{\delta}, \quad k=1,2,3 .
$$

Proof. I. It follows from (2.15) that

$$
b^{\delta}(s, y)+a^{\delta}(s, y) \partial_{y} \phi_{1}^{\delta}(s, y)=p^{\delta}(s) .
$$

Since $\phi_{1}^{\delta}(\cdot)$ is $[0,1]^{2}$ periodic, we get that

$$
\int_{0}^{1} \frac{p^{\delta}(s)-b^{\delta}(s, y)}{a^{\delta}(s, y)} \mathrm{d} y=\int_{0}^{1} \partial_{y} \phi_{1}^{\delta}(s, y) \mathrm{d} y=0, \quad s \in[0,1] .
$$

Solve $p^{\delta}(\cdot)$ from the above we get

$$
\partial_{y} \phi_{1}(s, y)=\left\{\begin{array}{lc}
\frac{b_{3}-b_{1}}{(1-\delta) a_{3}+\delta a_{1}} \delta, & (s, y) \in(\delta, 1) \times(\delta, 1), \\
\frac{b_{4}-b_{2}}{(1-\delta) a_{4}+\delta a_{2}} \delta, & (s, y) \in(0, \delta) \times(\delta, 1), \\
-\frac{b_{3}-b_{1}}{(1-\delta) a_{3}+\delta a_{1}}(1-\delta), & (s, y) \in(\delta, 1) \times(0, \delta), \\
-\frac{b_{4}-b_{2}}{(1-\delta) a_{4}+\delta a_{2}}(1-\delta), & (s, y) \in(0, \delta) \times(0, \delta) .
\end{array}\right.
$$


Thus

A direct calculation shows that

$$
\left|\partial_{y} \phi_{1}^{\delta}(s, y)\right| \leq\left\{\begin{array}{l}
\frac{2 \Lambda}{\lambda} \delta, \quad(s, y) \in[0,1] \times(\delta, 1), \\
\frac{2 \Lambda}{\lambda}(1-\delta),(s, y) \in[0,1] \times(0, \delta) .
\end{array}\right.
$$

$$
\begin{array}{r}
\left|\frac{1}{\delta} \int_{0}^{1} \mathrm{~d} y \int_{0}^{1} c^{\delta}(s, y) \partial_{y} \phi_{1}^{\delta}(s, y) \mathrm{d} s+\frac{\left(b_{3}-b_{1}\right)\left(c_{3}-c_{1}\right)}{a_{3}}\right|=\left|\delta(2-\delta) \frac{\left(b_{3}-b_{1}\right)\left(c_{3}-c_{1}\right)}{(1-\delta) a_{3}+\delta a_{1}}-\delta(1-\delta) \frac{\left(b_{4}-b_{2}\right)\left(c_{4}-c_{2}\right)}{(1-\delta) a_{4}+\delta a_{2}}\right| \\
\leq \delta(2-\delta) \frac{4 \Lambda^{2}}{\lambda}+\delta(1-\delta) \frac{4 \Lambda^{2}}{\lambda} \leq \frac{12 \Lambda^{2}}{\lambda} \delta
\end{array}
$$

II. It follows from $(2.16)$ and the periodicity of $\phi_{2}^{\delta}(\cdot)$ that

$$
\begin{aligned}
0 & =\int_{0}^{1} \int_{0}^{1}\left[b^{\delta}(s, y) \partial_{y} \phi_{2}^{\delta}(s, y)+a^{\delta}(s, y)\left|\partial_{y} \phi_{2}^{\delta}(s, y)\right|^{2}\right] \mathrm{d} s \mathrm{~d} y \\
& =\int_{0}^{1} \int_{0}^{1}\left[\left(b^{\delta}(s, y)-b_{1}\right) \partial_{y} \phi_{2}^{\delta}(s, y)+a^{\delta}(s, y)\left|\partial_{y} \phi_{2}^{\delta}(s, y)\right|^{2}\right] \mathrm{d} s \mathrm{~d} y
\end{aligned}
$$

Thus,

$$
\begin{aligned}
\int_{0}^{1} \int_{0}^{1}\left|\partial_{y} \phi_{2}^{\delta}(s, y)\right|^{2} \mathrm{~d} s \mathrm{~d} y & \leq \frac{1}{\lambda} \int_{0}^{1} \int_{0}^{1} a^{\delta}(s, y)\left|\partial_{y} \phi_{2}^{\delta}(s, y)\right|^{2} \mathrm{~d} s \mathrm{~d} y \\
& =-\frac{1}{\lambda} \int_{0}^{1} \int_{0}^{1}\left(b^{\delta}(s, y)-b_{1}\right) \partial_{y} \phi_{2}^{\delta}(s, y) \mathrm{d} s \mathrm{~d} y \\
& \leq \frac{1}{\lambda}\left(\int_{0}^{1} \int_{0}^{1}\left|b^{\delta}(s, y)-b_{1}\right|^{2} \mathrm{~d} s \mathrm{~d} y\right)^{\frac{1}{2}}\left(\int_{0}^{1} \int_{0}^{1}\left|\partial_{y} \phi_{2}^{\delta}(s, y)\right|^{2} \mathrm{~d} s \mathrm{~d} y\right)^{\frac{1}{2}} \\
& \leq \frac{2 \sqrt{2} \Lambda \sqrt{\delta}}{\lambda}\left(\int_{0}^{1} \int_{0}^{1}\left|\partial_{y} \phi_{2}^{\delta}(s, y)\right|^{2} \mathrm{~d} s \mathrm{~d} y\right)^{\frac{1}{2}}
\end{aligned}
$$

Therefore,

On the other hand, (2.20) implies

$$
\left\|\partial_{y} \phi_{2}^{\delta}\right\|_{L^{2}\left([0,1]^{2}\right)} \leq \frac{2 \sqrt{2} \Lambda \sqrt{\delta}}{\lambda} .
$$

$$
\left\|\partial_{y} \phi_{1}^{\delta}\right\|_{L^{2}\left([0,1]^{2}\right)} \leq \frac{2 \Lambda \sqrt{\delta}}{\lambda}
$$

Denote $\Phi^{\delta}(\cdot)=\phi_{2}^{\delta}(\cdot)-\phi_{1}^{\delta}(\cdot)$, we have

$$
\left\|\partial_{y} \Phi^{\delta}\right\|_{L^{2}\left([0,1]^{2}\right)} \leq \frac{2(1+\sqrt{2}) \Lambda \sqrt{\delta}}{\lambda} .
$$

For any $\varphi(\cdot) \in W_{\#}^{1,2}(0,1)$, it follows from $(2.15)-(2.16)$ that

$$
\begin{aligned}
& \int_{0}^{1}\left[b^{\delta}(s, y)+a^{\delta}(s, y) \partial_{y} \phi_{1}^{\delta}(s, y)\right] \partial_{y} \varphi(y) \mathrm{d} y=0, \quad \forall s \in[0,1] \\
& \int_{0}^{1} \int_{0}^{1}\left[b^{\delta}(s, y)+a^{\delta}(s, y) \partial_{y} \phi_{2}^{\delta}(s, y)\right] \partial_{y} \varphi(y) \mathrm{d} s \mathrm{~d} y=0 .
\end{aligned}
$$


Thus,

$$
\int_{0}^{1} \int_{0}^{1} a^{\delta}(s, y) \partial_{y} \Phi^{\delta}(s, y) \partial_{y} \varphi(y) \mathrm{d} s \mathrm{~d} y=0 .
$$

Therefore $\int_{0}^{1} a^{\delta}(s, y) \partial_{y} \Phi^{\delta}(s, y) \mathrm{d} s$ is a constant and consequently,

$$
\begin{aligned}
\left|\int_{0}^{1} a^{\delta}(s, y) \partial_{y} \Phi^{\delta}(s, y) \mathrm{d} s\right| & =\left|\int_{0}^{1} \int_{0}^{1} a^{\delta}(s, w) \partial_{y} \Phi^{\delta}(s, x) \mathrm{d} s \mathrm{~d} x\right| \\
& =\left|\int_{0}^{1} \int_{0}^{1}\left(a^{\delta}(s, x)-a_{1}\right) \partial_{y} \Phi^{\delta}(s, x) \mathrm{d} s \mathrm{~d} x\right| \\
& \leq\left(\int_{0}^{1} \int_{0}^{1}\left|a^{\delta}(s, x)-a_{1}\right|^{2} \mathrm{~d} s \mathrm{~d} x\right)^{\frac{1}{2}}\left\|\partial_{y} \Phi^{\delta}\right\|_{L^{2}\left([0,1]^{2}\right)} \\
& \leq \sqrt{2} \Lambda \sqrt{\delta} \cdot \frac{2(1+\sqrt{2}) \Lambda}{\lambda} \sqrt{\delta}=\frac{2(2+\sqrt{2}) \Lambda^{2}}{\lambda} \delta, \quad \forall y \in[0,1] .
\end{aligned}
$$

Nothing that (2.15) implies

$$
\int_{0}^{1}\left[b^{\delta}(s, y) \partial_{y} \phi_{2}^{\delta}(s, y)+a^{\delta}(s, y) \partial_{y} \phi_{1}^{\delta}(s, y) \partial_{y} \phi_{2}^{\delta}(s, y)\right] \mathrm{d} y=0, \quad \forall s \in[0,1],
$$

we get from (2.22) that

$$
\int_{0}^{1} \int_{0}^{1} a^{\delta}(s, y) \partial_{y} \Phi^{\delta}(s, y) \partial_{y} \phi_{2}^{\delta}(s, y) \mathrm{d} s \mathrm{~d} y=0
$$

Thus denote

$$
\theta_{1, \delta}=\frac{b_{3}-b_{1}}{(1-\delta) a_{3}+\delta a_{1}}, \quad \theta_{2, \delta}=\frac{b_{4}-b_{2}}{(1-\delta) a_{4}+\delta a_{2}},
$$

we have

$$
\begin{aligned}
\left\|\partial_{y} \Phi^{\delta}\right\|_{L^{2}\left([0,1]^{2} ; a^{\delta}\right)}^{2} \equiv & \int_{0}^{1} \int_{0}^{1} a^{\delta}(s, y)\left|\partial_{y} \Phi^{\delta}(s, y)\right|^{2} \mathrm{~d} s \mathrm{~d} y=-\int_{0}^{1} \int_{0}^{1} a^{\delta}(s, y) \partial_{y} \Phi^{\delta}(s, y) \partial_{y} \phi_{1}^{\delta}(s, y) \mathrm{d} s \mathrm{~d} y \\
= & -\int_{\delta}^{1} \int_{\delta}^{1} a^{\delta}(s, y) \partial_{y} \Phi^{\delta}(s, y) \theta_{1, \delta} \delta \mathrm{d} s \mathrm{~d} y-\int_{\delta}^{1} \int_{0}^{\delta} a^{\delta}(s, y) \partial_{y} \Phi^{\delta}(s, y) \theta_{2, \delta} \delta \mathrm{d} s \mathrm{~d} y \\
& +\int_{0}^{\delta} \int_{0}^{1} a^{\delta}(s, y) \partial_{y} \Phi^{\delta}(s, y) \theta_{1, \delta}(1-\delta) \mathrm{d} s \mathrm{~d} y \\
& +\int_{0}^{\delta} \int_{0}^{\delta} a^{\delta}(s, y) \partial_{y} \Phi^{\delta}(s, y)\left(\theta_{2, \delta}-\theta_{1, \delta}\right)(1-\delta) \mathrm{d} s \mathrm{~d} y \\
\leq & \int_{\delta}^{1} \int_{0}^{1} a^{\delta}(s, y)\left|\partial_{y} \Phi^{\delta}(s, y)\right| \cdot \frac{2 \Lambda}{\lambda} \delta \mathrm{d} s \mathrm{~d} y+\int_{0}^{\delta}\left|\int_{0}^{1} a^{\delta}(s, y) \partial_{y} \Phi^{\delta}(s, y) \mathrm{d} s\right| \cdot \frac{2 \Lambda}{\lambda}(1-\delta) \mathrm{d} y \\
& +\int_{0}^{\delta} \int_{0}^{\delta} a^{\delta}(s, y)\left|\partial_{y} \Phi^{\delta}(s, y)\right| \cdot \frac{4 \Lambda}{\lambda}(1-\delta) \mathrm{d} s \mathrm{~d} y \\
\leq & \frac{2 \Lambda}{\lambda} \delta\left\|\partial_{y} \Phi^{\delta}\right\|_{L^{2}\left([0,1]^{2} ; a^{\delta}\right)} \cdot \sqrt{\Lambda} \\
& +\frac{2(2+\sqrt{2}) \Lambda^{2}}{\lambda} \delta \cdot \frac{2 \Lambda}{\lambda} \delta+\frac{4 \Lambda}{\lambda} \cdot \sqrt{\Lambda} \delta\left(\int_{0}^{\delta} \int_{0}^{\delta} a^{\delta}(s, y)\left|\partial_{y} \Phi^{\delta}(s, y)\right|^{2} \mathrm{~d} s \mathrm{~d} y\right)^{\frac{1}{2}} \\
\leq & \frac{6 \Lambda \sqrt{\Lambda}}{\lambda} \delta\left\|\partial_{y} \Phi^{\delta}\right\|_{L^{2}\left([0,1]^{2} ; a^{\delta}\right)}+\frac{4(2+\sqrt{2}) \Lambda^{3}}{\lambda^{2}} \delta^{2} .
\end{aligned}
$$


Thus

$$
\left\|\partial_{y} \Phi^{\delta}\right\|_{L^{2}\left([0,1]^{2}\right)} \leq \frac{1}{\sqrt{\lambda}}\left\|\partial_{y} \Phi^{\delta}\right\|_{L^{2}\left([0,1]^{2} ; a^{\delta}\right)}^{2} \leq \frac{8 \Lambda \sqrt{\Lambda}}{\lambda \sqrt{\lambda}} \delta .
$$

Then, (2.30) can be improved as

$$
\left|\int_{0}^{1} a^{\delta}(s, y) \partial_{y} \Phi^{\delta}(s, y) \mathrm{d} s\right| \leq \sqrt{2} \Lambda \sqrt{\delta} \cdot \frac{8 \Lambda \sqrt{\Lambda}}{\lambda \sqrt{\lambda}} \delta=\frac{12 \Lambda^{2} \sqrt{\Lambda}}{\lambda \sqrt{\lambda}} \delta \sqrt{\delta}, \quad \forall y \in[0,1] .
$$

Moreover,

$$
\begin{aligned}
\left(\int_{0}^{1}\left|\int_{0}^{1} \partial_{y} \Phi^{\delta}(s, y) \mathrm{d} s\right|^{2} \mathrm{~d} y\right)^{\frac{1}{2}} & \leq \frac{1}{\lambda}\left(\int_{0}^{1}\left|\int_{0}^{1}\left(a_{1} \chi_{[\delta, 1]}(y)+a_{3} \chi_{(0, \delta)}(y)\right) \partial_{y} \Phi^{\delta}(s, y) \mathrm{d} s\right|^{2} \mathrm{~d} y\right)^{\frac{1}{2}} \\
\leq & \frac{1}{\lambda}\left(\int_{0}^{1}\left|\int_{0}^{1} a^{\delta}(s, y) \partial_{y} \Phi^{\delta}(s, y) \mathrm{d} s\right|^{2} \mathrm{~d} y\right)^{\frac{1}{2}} \\
& +\frac{1}{\lambda}\left(\int_{0}^{1}\left|\int_{0}^{\delta}\left(\left(a_{2}-a_{1}\right) \chi_{[\delta, 1]}(y)+\left(a_{4}-a_{3}\right) \chi_{(0, \delta)}(y)\right) \partial_{y} \Phi^{\delta}(s, y) \mathrm{d} s\right|^{2} \mathrm{~d} y\right)^{\frac{1}{2}} \\
& \frac{12 \Lambda^{2} \sqrt{\Lambda}}{\lambda^{2} \sqrt{\lambda}} \delta \sqrt{\delta}+\frac{1}{\lambda}\left[\int_{0}^{1}\left(4 \Lambda^{2} \delta \int_{0}^{\delta}\left|\partial_{y} \Phi^{\delta}(s, y)\right|^{2} \mathrm{~d} s\right) \mathrm{d} y\right]^{\frac{1}{2}} \\
& \frac{12 \Lambda^{2} \sqrt{\Lambda}}{\lambda^{2} \sqrt{\lambda}} \delta \sqrt{\delta}+\frac{2 \Lambda \sqrt{\delta}}{\lambda} \cdot \frac{8 \Lambda \sqrt{\Lambda}}{\lambda \sqrt{\lambda}} \delta=\frac{28 \Lambda^{2} \sqrt{\Lambda}}{\lambda^{2} \sqrt{\lambda}} \delta \sqrt{\delta} .
\end{aligned}
$$

Therefore,

$$
\begin{aligned}
\mid \frac{1}{\delta} \int_{0}^{1} \int_{0}^{1} c^{\delta}(s, y) \partial_{y} \phi_{2}^{\delta}(s, y) \mathrm{d} s \mathrm{~d} y & \frac{\left(b_{3}-b_{1}\right)\left(c_{3}-c_{1}\right)}{a_{3}} \mid \\
\leq & \left|\frac{1}{\delta} \int_{0}^{1} \mathrm{~d} y \int_{0}^{1} c^{\delta}(s, y) \partial_{y} \phi_{1}^{\delta}(s, y) \mathrm{d} s+\frac{\left(b_{3}-b_{1}\right)\left(c_{3}-c_{1}\right)}{a_{3}}\right| \\
& +\left|\frac{1}{\delta} \int_{0}^{1} \int_{0}^{1} c^{\delta}(s, y) \partial_{y} \Phi^{\delta}(s, y) \mathrm{d} s \mathrm{~d} y\right| \\
\leq & \frac{12 \Lambda^{2}}{\lambda} \delta+\left|\frac{1}{\delta} \int_{0}^{1} \int_{0}^{1}\left(c^{\delta}(s, y)-c_{1} \chi_{[\delta, 1]}(y)-c_{3} \chi_{(0, \delta)}(y)\right) \partial_{y} \Phi^{\delta}(s, y) \mathrm{d} s \mathrm{~d} y\right| \\
& +\left|\frac{1}{\delta} \int_{0}^{1} \int_{0}^{1}\left(c_{1} \chi_{[\delta, 1]}(y)+c_{3} \chi_{(0, \delta)}(y)\right) \partial_{y} \Phi^{\delta}(s, y) \mathrm{d} s \mathrm{~d} y\right| \\
= & \frac{12 \Lambda^{2}}{\lambda} \delta+\left|\frac{1}{\delta} \int_{0}^{1} \int_{0}^{\delta}\left(\left(c_{2}-c_{1}\right) \chi_{[\delta, 1]}(y)+\left(c_{4}-c_{3}\right) \chi_{(0, \delta)}(y)\right) \partial_{y} \Phi^{\delta}(s, y) \mathrm{d} s \mathrm{~d} y\right| \\
& +\left|\frac{1}{\delta} \int_{0}^{1}\left(\int_{0}^{1} \partial_{y} \Phi^{\delta}(s, y) \mathrm{d} s\right)\left(c_{1} \chi_{[\delta, 1]}(y)+c_{3} \chi_{(0, \delta)}(y)\right) \mathrm{d} y\right| \\
\leq & \frac{12 \Lambda^{2}}{\lambda} \delta+\frac{2 \Lambda \sqrt{\delta}}{\delta}\left\|\partial_{y} \Phi^{\delta}\right\|_{L^{2}\left([0,1]^{2}\right)}+\frac{\Lambda}{\delta}\left(\int_{0}^{1}\left|\int_{0}^{1} \partial_{y} \Phi^{\delta}(s, y) \mathrm{d} s\right|^{2} \mathrm{~d} y\right)^{\frac{1}{2}} \\
\leq & \frac{12 \Lambda^{2}}{\lambda} \delta+\frac{2 \Lambda \sqrt{\delta}}{\delta} \cdot \frac{8 \Lambda \sqrt{\Lambda}}{\lambda \sqrt{\lambda}} \delta+\frac{\Lambda}{\delta} \cdot \frac{28 \Lambda^{2} \sqrt{\Lambda}}{\lambda^{2} \sqrt{\lambda}} \delta \sqrt{\delta} \leq \frac{56 \Lambda^{3} \sqrt{\Lambda}}{\lambda^{2} \sqrt{\lambda}} \sqrt{\delta} .
\end{aligned}
$$


III. By (2.17),

$$
\int_{0}^{1} b^{\delta}(s, y) \mathrm{d} s+\int_{0}^{1} a^{\delta}(s, y) \mathrm{d} s \partial_{y} \phi_{3}^{\delta}(y)
$$

is a constant. Then similar to $(2.19)$, we can get from the periodicity of $\phi_{3}^{\delta}(\cdot)$ that

$$
\partial_{y} \phi_{3}^{\delta}(y)= \begin{cases}\frac{\left(b_{3}-b_{1}\right)(1-\delta)+\left(b_{4}-b_{2}\right) \delta}{\delta(1-\delta) a_{1}+\delta^{2} a_{2}+(1-\delta)^{2} a_{3}+\delta(1-\delta) a_{4}} \delta, & y \in(\delta, 1), \\ -\frac{\left(b_{3}-b_{1}\right)(1-\delta)+\left(b_{4}-b_{2}\right) \delta}{\delta(1-\delta) a_{1}+\delta^{2} a_{2}+(1-\delta)^{2} a_{3}+\delta(1-\delta) a_{4}}(1-\delta), & y \in(0, \delta) .\end{cases}
$$

Thus

$$
\frac{1}{\delta} \int_{0}^{1} \int_{0}^{1} c^{\delta}(s, y) \partial_{y} \phi_{3}^{\delta}(s, y) \mathrm{d} s \mathrm{~d} y=-\frac{(1-\delta)\left[(1-\delta)\left(b_{3}-b_{1}\right)+\delta\left(b_{4}-b_{2}\right)\right] \cdot\left[(1-\delta)\left(c_{3}-c_{1}\right)+\delta\left(c_{4}-c_{2}\right)\right]}{\delta(1-\delta) a_{1}+\delta^{2} a_{2}+(1-\delta)^{2} a_{3}+\delta(1-\delta) a_{4}} .
$$

One can verify that

$$
\begin{aligned}
\left|\frac{1}{\delta} \int_{0}^{1} \int_{0}^{1} c^{\delta}(s, y) \partial_{y} \phi_{3}^{\delta}(s, y) \mathrm{d} s \mathrm{~d} y+\frac{\left(b_{3}-b_{1}\right)\left(c_{3}-c_{1}\right)}{a_{3}}\right| & \leq \frac{12 \Lambda^{2}}{\lambda} \delta+\mid \frac{\left(b_{3}-b_{1}\right)\left(c_{3}-c_{1}\right)}{a_{3}} \\
& -\frac{(1-\delta)^{2}\left(b_{3}-b_{1}\right)\left(c_{3}-c_{1}\right)}{\delta(1-\delta) a_{1}+\delta^{2} a_{2}+(1-\delta)^{2} a_{3}+\delta(1-\delta) a_{4}} \mid \\
& \leq \frac{12 \Lambda^{2}}{\lambda} \delta+\frac{8 \Lambda^{3}}{\lambda^{2}} \delta \leq \frac{20 \Lambda^{3}}{\lambda^{2}} \delta .
\end{aligned}
$$

Combining (2.21), (2.35) and (2.37), we get (2.18) with $C=\frac{56 \Lambda^{3} \sqrt{\Lambda}}{\lambda^{2} \sqrt{\lambda}}$.

The following result is concerned with the well-posedness and regularity of state equation (1.1).

Lemma 2.3. Let (S1)-(S4) hold and $z_{0} \in L^{\infty}(\Omega)$. Then for any $u(\cdot) \in \mathcal{U},(1.1)$ admits a unique weak solution $z(\cdot) \in L^{2}\left(0, T ; H_{0}^{1}(\Omega)\right) \cap L^{\infty}\left(\Omega_{T}\right)$. Furthermore, there exist a constant $K>0$, independent of $u(\cdot) \in \mathcal{U}$, such that

$$
\|z(\cdot)\|_{L^{2}\left(0, T ; H_{0}^{1}(\Omega)\right)}+\|z(\cdot)\|_{L^{\infty}\left(\Omega_{T}\right)} \leq K .
$$

Moreover, there exists an $\alpha \in(0,1)$, such that for any $Q_{0} \subset \subset \Omega_{T}$, it holds that

$$
\|z(\cdot)\|_{C^{\alpha}\left(Q_{0}\right)} \leq C\left(Q_{0}\right)
$$

for some constant $C\left(Q_{0}\right)$.

Proof. The result is quite standard. We give a sketch of the proof. Fix $u(\cdot) \in \mathcal{U}$. Let $m>0$, define

$$
f^{m}(t, x, z, u)= \begin{cases}f(t, x, z, u), & |z| \leq m \\ f(t, x,-m, u), & z \leq-m \\ f(t, x, m, u), & z \geq m\end{cases}
$$

For fixed $z(\cdot) \in L^{2}\left(\Omega_{T}\right)$, let $z^{m}(\cdot)$ be the solution of

$$
\left\{\begin{array}{l}
\partial_{t} z^{m}(t, x)-\nabla \cdot\left(A(t, x, u(t, x)) \nabla z^{m}(t, x)\right)=f^{m}(t, x, z(t, x), u(t, x)), \quad \text { in } \Omega_{T}, \\
z^{m}(t, x)=0, \quad \text { on }[0, T] \times \partial \Omega, \\
z^{m}(0, x)=z_{0}(x), \quad \text { in } \Omega,
\end{array}\right.
$$


Then there exist a constant $C_{m}>0$ such that

$$
\left\|z^{m}(\cdot)\right\|_{L^{2}\left(0, T ; H_{0}^{1}(\Omega)\right)}+\left\|z^{m}(\cdot)\right\|_{L^{\infty}\left(\Omega_{T}\right)} \leq C_{m}
$$

Moreover, there exists an $\beta=\beta_{m} \in(0,1)$, such that for any $Q_{0} \subset \subset \Omega_{T}$, it holds that

$$
\left\|z^{m}(\cdot)\right\|_{C^{\beta}\left(Q_{0}\right)} \leq C_{m}\left(Q_{0}\right)
$$

for some constant $C_{m}\left(Q_{0}\right)$.

Using (2.40)-(2.41), we can see that the map $z(\cdot) \mapsto z^{m}(\cdot)$ is continuous and compact from some ball of $L^{2}\left(\Omega_{T}\right)$ to itself. Thus, Schauder fixed point theorem implies that the map has a fixed point $Z^{m}(\cdot)$. We have $Z^{m}(\cdot) \in L^{2}\left(0, T ; H_{0}^{1}(\Omega)\right)$ and

$$
\left\{\begin{array}{l}
\partial_{t} Z^{m}(t, x)-\nabla \cdot\left(A(t, x, u(t, x)) \nabla Z^{m}(t, x)\right)=f^{m}\left(t, x, Z^{m}(t, x), u(t, x)\right), \quad \text { in } \Omega_{T}, \\
Z^{m}(t, x)=0, \quad \text { on }[0, T] \times \partial \Omega, \\
Z^{m}(0, x)=z_{0}(x), \quad \text { in } \Omega .
\end{array}\right.
$$

Noting that (S4) holds, we can modify the proof of Theorem 7.1 of Chapter 3 in [9] to get that

$$
\left\|Z^{m}(\cdot)\right\|_{L^{\infty}\left(\Omega_{T}\right)} \leq C
$$

with $C$ being independent of $m$. Let $m>C$, we see that (1.1) admits a unique weak solution $z(\cdot) \in$ $L^{2}\left(0, T ; H_{0}^{1}(\Omega)\right) \cap L^{\infty}\left(\Omega_{T}\right)$ and $(2.38)$ holds. Finally, by $(1.6)$,

$$
|f(t, x, z(t, x), u(t, x))| \leq M_{K}
$$

Thus, (2.39) follows from Theorem 10.1 of Chapter 3 in [9].

Lemma 2.4. Let $\delta \in(0,1), r>0$,

$$
\zeta_{m}(t, x)=\left\{\begin{array}{ll}
\delta_{m 1}, & \text { if }\left(\{s\},\left\{x_{1}\right\}\right) \in[\delta, 1) \times[\delta, 1), \\
\delta_{m 2}, & \text { if }\left(\{s\},\left\{x_{1}\right\}\right) \in[0, \delta) \times[\delta, 1), \\
\delta_{m 3}, & \text { if }\left(\{s\},\left\{x_{1}\right\}\right) \in[\delta, 1) \times[0, \delta), \\
\delta_{m 4}, & \text { if }\left(\{s\},\left\{x_{1}\right\}\right) \in[0, \delta) \times[0, \delta),
\end{array} \quad(t, x) \in \Omega_{T} .\right.
$$

Then $\zeta_{m}\left(\frac{t}{\varepsilon^{r}}, \frac{x_{1}}{\varepsilon}\right)(m=1,2,3,4)$ converges weakly to $\mu_{m}$ in $L^{2}\left(\Omega_{T}\right)$ with

$$
\mu_{1}=(1-\delta)^{2}, \quad \mu_{2}=\mu_{3}=\delta(1-\delta), \quad \mu_{4}=\delta^{2}
$$

Proof. Such results are quite well-known and can be proved by modifying the proof of Riemann's lemma. One can verify easily that for any rectangle $F \subset \Omega_{T}$,

$$
\lim _{\varepsilon \rightarrow 0^{+}} \int_{\Omega_{T}} \chi_{F}(t, x) \zeta_{1}\left(\frac{t}{\varepsilon^{r}}, \frac{x_{1}}{\varepsilon}\right) \mathrm{d} t \mathrm{~d} x=\mu_{1} \int_{\Omega_{T}} \chi_{F}(t, x) \mathrm{d} t \mathrm{~d} x .
$$

Since the set of all linear combinations of characteristic functions $\chi_{F}(\cdot)$ is dense in $L^{2}\left(\Omega_{T}\right)$, we get that $\zeta_{1}\left(\frac{t}{\varepsilon^{r}}\right.$, $\left.\frac{x_{1}}{\varepsilon}\right)$ converges weakly to $\mu_{1}$ in $L^{2}\left(\Omega_{T}\right)$. The remains are similar. 
Lemma 2.5. Let $n \geq 2$. Let $\xi, \eta \in \mathbb{R}^{n}$ be two nonzero vectors. Then

$$
\sup _{|x|=1} \xi^{\top} x x^{\top} \eta=\frac{|\xi||\eta|+\xi^{\top} \eta}{2}
$$

where $E^{\top}$ denotes the transpose of a matrix $E$.

The proof of above lemma is easy. See [11], for example.

\section{Proof of the MAIN THEOREM}

In this section, we present a proof of our main theorem. The proof is divided into several steps. Let $\bar{u}(\cdot) \in \mathcal{U}$ be an optimal control and $\bar{z}(\cdot)$ be the corresponding optimal state. Let $r>0, u_{2}(\cdot), u_{3}(\cdot), u_{4}(\cdot) \in \mathcal{U}$ be fixed.

\subsection{Homogenizing spike variation of the control}

Let $\delta \in(0,1)$ and $\varepsilon>0$. For any $(t, x)=\left(t, x_{1}, x_{2}, \ldots, x_{n}\right) \in \Omega_{T}$, define

$$
u^{\delta, \varepsilon}(t, x)= \begin{cases}\bar{u}(t, x), & \text { if }\left(\left\{\frac{t}{\varepsilon^{r}}\right\},\left\{\frac{x_{1}}{\varepsilon}\right\}\right) \in[\delta, 1) \times[\delta, 1), \\ u_{2}(t, x), & \text { if }\left(\left\{\frac{t}{\varepsilon^{r}}\right\},\left\{\frac{x_{1}}{\varepsilon}\right\}\right) \in[0, \delta) \times[\delta, 1), \\ u_{3}(t, x), & \text { if }\left(\left\{\frac{t}{\varepsilon^{r}}\right\},\left\{\frac{x_{1}}{\varepsilon}\right\}\right) \in[\delta, 1) \times[0, \delta), \\ u_{4}(t, x), & \text { if }\left(\left\{\frac{t}{\varepsilon^{r}}\right\},\left\{\frac{x_{1}}{\varepsilon}\right\}\right) \in[0, \delta) \times[0, \delta) .\end{cases}
$$

Then $u^{\delta, \varepsilon}(\cdot) \in \mathcal{U}$. Let $z^{\delta, \varepsilon}(\cdot)$ be the state corresponding to $u^{\delta, \varepsilon}(\cdot)$, i.e.,

$$
\left\{\begin{array}{l}
\partial_{t} z^{\delta, \varepsilon}(t, x)-\nabla \cdot\left(A\left(t, x, u^{\delta, \varepsilon}(t, x)\right) \nabla z^{\delta, \varepsilon}(t, x)\right)=f\left(t, x, z^{\delta, \varepsilon}(t, x), u^{\delta, \varepsilon}(t, x)\right), \quad \text { in } \Omega_{T}, \\
z^{\delta, \varepsilon}(t, x)=0, \quad \text { on }[0, T] \times \partial \Omega, \\
z^{\delta, \varepsilon}(0, x)=z_{0}(x), \quad \text { in } \Omega .
\end{array}\right.
$$

By Lemma 2.3, there exists constants $K>0$ and $\alpha \in(0,1)$, independent of $\delta, \varepsilon$, such that

$$
\left\|z^{\delta, \varepsilon}(\cdot)\right\|_{L^{2}\left(0, T ; H_{0}^{1}(\Omega)\right)}+\left\|z^{\delta, \varepsilon}(\cdot)\right\|_{L^{\infty}\left(\Omega_{T}\right)} \leq K
$$

and

$$
\left\|z^{\delta, \varepsilon}\right\|_{C^{\alpha}\left(Q_{0}\right)} \leq C\left(Q_{0}\right)
$$

for any $Q_{0} \subset \subset \Omega_{T}$ with some constant $C\left(Q_{0}\right)$.

By (3.3), for fixed $\delta \in(0,1)$, we can extract a subsequence (still denoted by itself) such that $z^{\delta, \varepsilon}(\cdot)$ converges to a function $z^{\delta}(\cdot)$ weakly in $L^{2}\left(0, T ; H_{0}^{1}(\Omega)\right)$ as $\varepsilon \rightarrow 0^{+}$. By (3.4) and Arzelá-Ascoli's theorem, $z^{\delta, \varepsilon}(\cdot)$ converges uniformly to $z^{\delta}(\cdot)$ in $C\left(Q_{0}\right)$ for any $Q_{0} \subset \subset \Omega_{T}$. Then, it follows easily from

$$
\left\|z^{\delta, \varepsilon}(\cdot)\right\|_{L^{\infty}\left(\Omega_{T}\right)} \leq K
$$

that $z^{\delta, \varepsilon}(\cdot)$ converges strongly to $z^{\delta}(\cdot)$ in $L^{2}\left(\Omega_{T}\right)$ and almost everywhere in $\Omega_{T}$.

By (1.6) and (3.3),

$$
\left|f\left(t, x, z^{\delta, \varepsilon}(t, x), u^{\delta, \varepsilon}(t, x)\right)-f\left(t, x, z^{\delta}(t, x), u^{\delta, \varepsilon}(t, x)\right)\right| \leq M_{K}\left|z^{\delta, \varepsilon}(t, x)-z^{\delta}(x)\right| .
$$


On the other hand, by Lemma 2.4 , for any $h \in L^{2}\left(\Omega_{T}\right)$, when $\varepsilon \rightarrow 0^{+}$,

$$
\begin{array}{r}
\int_{\Omega_{T}} f\left(t, x, z^{\delta}(t, x), u^{\delta, \varepsilon}(t, x)\right) h(t, x) \mathrm{d} t \mathrm{~d} x \rightarrow(1-\delta)^{2} \int_{\Omega_{T}} f\left(t, x, z^{\delta}(t, x), \bar{u}(t, x)\right) h(t, x) \mathrm{d} t \mathrm{~d} x \\
+\delta(1-\delta) \int_{\Omega_{T}} f\left(t, x, z^{\delta}(t, x), u_{2}(t, x)\right) h(t, x) \mathrm{d} t \mathrm{~d} x+\delta(1-\delta) \int_{\Omega_{T}} f\left(t, x, z^{\delta}(t, x), u_{3}(t, x)\right) h(t, x) \mathrm{d} t \mathrm{~d} x \\
+\delta^{2} \int_{\Omega_{T}} f\left(t, x, z^{\delta}(t, x), u_{4}(t, x)\right) h(t, x) \mathrm{d} t \mathrm{~d} x
\end{array}
$$

Combing the above with (3.5), we get that along a subsequence $\varepsilon \rightarrow 0^{+}$,

$$
\begin{aligned}
f\left(t, x, z^{\delta, \varepsilon}(t, x), u^{\delta, \varepsilon}(t, x)\right) & (1-\delta)^{2} f\left(t, x, z^{\delta}(t, x), \bar{u}(t, x)\right)+\delta(1-\delta) f\left(t, x, z^{\delta}(t, x), u_{2}(t, x)\right) \\
& +\delta(1-\delta) f\left(t, x, z^{\delta}(t, x), u_{3}(t, x)\right)+\delta^{2} f\left(t, x, z^{\delta}(t, x), u_{4}(t, x)\right), \text { weakly in } L^{2}\left(\Omega_{T}\right) .
\end{aligned}
$$

Let

$$
\begin{aligned}
h^{\delta, \varepsilon}(t, x)= & f\left(t, x, z^{\delta, \varepsilon}(t, x), u^{\delta, \varepsilon}(t, x)\right)-(1-\delta)^{2} f\left(t, x, z^{\delta}(t, x), \bar{u}(t, x)\right)-\delta(1-\delta) f\left(t, x, z^{\delta}(t, x), u_{2}(t, x)\right) \\
& -\delta(1-\delta) f\left(t, x, z^{\delta}(t, x), u_{3}(t, x)\right) h(t, x)-\delta^{2} f\left(t, x, z^{\delta}(t, x), u_{4}(t, x)\right)
\end{aligned}
$$

and $\tilde{z}^{\delta, \varepsilon}(\cdot)$ be the solution of

$$
\begin{cases}\partial_{t} \tilde{z}^{\delta, \varepsilon}(t, x)-\nabla \cdot\left(A\left(t, x, u^{\delta, \varepsilon}(t, x)\right) \nabla \tilde{z}^{\delta, \varepsilon}(t, x)\right)=h^{\delta, \varepsilon}(t, x), \quad \text { in } \Omega_{T} \\ \tilde{z}^{\delta, \varepsilon}(t, x)=0, & \text { on }[0, T] \times \partial \Omega, \\ \tilde{z}^{\delta, \varepsilon}(0, x)=0, & \text { in } \Omega .\end{cases}
$$

Then

$$
\int_{\Omega}\left|\tilde{z}^{\delta, \varepsilon}(T, x)\right|^{2} \mathrm{~d} x+\lambda \int_{\Omega_{T}}\left|\nabla \tilde{z}^{\delta, \varepsilon}(t, x)\right|^{2} \mathrm{~d} t \mathrm{~d} x \leq \int_{\Omega_{T}} \tilde{z}^{\delta, \varepsilon}(t, x) h^{\delta, \varepsilon}(t, x) \mathrm{d} t \mathrm{~d} x .
$$

As $z^{\delta, \varepsilon}(\cdot)$ converges strongly in $L^{2}\left(\Omega_{T}\right), \tilde{z}^{\delta, \varepsilon}(\cdot)$ converges strongly in $L^{2}\left(\Omega_{T}\right)$ too. Consequently, it follows from (3.7) that

$$
\tilde{z}^{\delta, \varepsilon}(\cdot) \rightarrow 0, \quad \text { strongly in } L^{2}\left(0, T ; H_{0}^{1}(\Omega)\right)
$$

By Lemma 2.1,

$$
z^{\delta, \varepsilon}(\cdot)-\tilde{z}^{\delta, \varepsilon}(\cdot) \rightarrow z^{\delta}(\cdot), \quad \text { weakly in } L^{2}\left(0, T ; H_{0}^{1}(\Omega)\right)
$$

with $z^{\delta}(\cdot)$ being the weak solution of

$$
\left\{\begin{array}{rc}
\partial_{t} z^{\delta}(t, x)-\nabla \cdot\left(Q^{\delta}(t, x) \nabla z^{\delta}(t, x)\right)= & (1-\delta)^{2} f\left(t, x, z^{\delta}(t, x), \bar{u}(t, x)\right) \\
& +\delta(1-\delta) f\left(t, x, z^{\delta}(t, x), u_{2}(t, x)\right) \\
& +\delta(1-\delta) f\left(t, x, z^{\delta}(t, x), u_{3}(t, x)\right) \\
& +\delta^{2} f\left(t, x, z^{\delta}(t, x), u_{4}(t, x)\right), \quad \text { in } \Omega_{T}, \\
z^{\delta}(t, x)=0, & \text { on }[0, T] \times \partial \Omega, \\
z^{\delta}(0, x)=z_{0}(x), & \text { in } \Omega,
\end{array}\right.
$$


where $Q^{\delta}(\cdot)=\left(q_{i j}^{\delta}(\cdot)\right) \in L^{\infty}\left(\Omega_{T} ; \mathcal{S}_{+}^{n}\right)$ is given by

$$
q_{i j}^{\delta}(t, x)=\int_{0}^{1} \mathrm{~d} y_{1} \int_{0}^{1}\left(g_{i j}^{\delta}\left(t, x, s, y_{1}\right)+g_{i 1}^{\delta}\left(t, x, s, y_{1}\right) \partial_{y_{1}} \varphi^{j}\left(t, x, s, y_{1}\right)\right) \mathrm{d} s, \quad 1 \leq i, j \leq n
$$

and

$$
\left(g_{i j}^{\delta}\left(t, x, s, y_{1}\right)\right)= \begin{cases}a_{i j}(t, x, \bar{u}(t, x)), & \text { if }\left(\{s\},\left\{y_{1}\right\}\right) \in[\delta, 1) \times[\delta, 1), \\ a_{i j}\left(t, x, u_{2}(t, x)\right), & \text { if }\left(\{s\},\left\{y_{1}\right\}\right) \in[0, \delta) \times[\delta, 1), \\ a_{i j}\left(t, x, u_{3}(t, x)\right), & \text { if }\left(\{s\},\left\{y_{1}\right\}\right) \in[\delta, 1) \times[0, \delta), \\ a_{i j}\left(t, x, u_{4}(t, x)\right), & \text { if }\left(\{s\},\left\{y_{1}\right\}\right) \in[0, \delta) \times[0, \delta) .\end{cases}
$$

For $r<2, \varphi^{k}(t, x, \cdot) \in L_{\#}^{2}\left(0,1 ; W_{\#}^{1,2}(0,1) / \mathbb{R}\right)$ is the unique solution of

$$
\partial_{y_{1}}\left(g_{1 k}^{\delta}\left(t, x, s, y_{1}\right)+g_{11}^{\delta}\left(t, x, s, y_{1}\right) \partial_{y_{1}} \varphi^{k}\left(t, x, s, y_{1}\right)\right)=0 .
$$

For $r=2, \varphi^{k}(t, x, \cdot)$ is the solution of

$$
\partial_{s} \varphi^{k}\left(t, x, s, y_{1}\right)-\partial_{y_{1}}\left(g_{1 k}^{\delta}\left(t, x, s, y_{1}\right)+g_{11}^{\delta}\left(t, x, s, y_{1}\right) \partial_{y_{1}} \varphi^{k}\left(t, x, s, y_{1}\right)\right)=0 .
$$

While for $r>2, \varphi^{k}(t, x, s, y)=\varphi^{k}\left(t, x, y_{1}\right)$ is the solution of

$$
\partial_{y_{1}}\left(\int_{0}^{1} g_{1 k}^{\delta}\left(t, x, s, y_{1}\right) \mathrm{d} s+\int_{0}^{1} g_{i j}^{\delta}\left(t, x, s, y_{1}\right) \mathrm{d} s \partial_{y_{1}} \varphi^{k}\left(t, x, y_{1}\right)\right)=0
$$

Combining (3.9) with (3.8), along a subsequence, we obtain

$$
z^{\delta, \varepsilon}(\cdot) \rightarrow z^{\delta}(\cdot), \quad \text { weakly in } L^{2}\left(0, T ; H_{0}^{1}(\Omega)\right)
$$

Note that for fixed $\delta \in(0,1)$, since any subsequence of $z^{\delta, \varepsilon}(\cdot)$ has a further subsequence converging to the same $z^{\delta}(\cdot)$ weakly in $L^{2}\left(0, T ; H_{0}^{1}(\Omega)\right), z^{\delta, \varepsilon}(\cdot)$ itself must converge to $z^{\delta}(\cdot)$ weakly in $L^{2}\left(0, T ; H_{0}^{1}(\Omega)\right)$.

In addition, by the optimality of $\bar{u}(\cdot)$, we have

$$
\begin{aligned}
J(\bar{u}(\cdot)) \leq & \lim _{\varepsilon \rightarrow 0^{+}} J\left(u^{\delta, \varepsilon}(\cdot)\right) \equiv J^{\delta}=\int_{\Omega_{T}}\left((1-\delta)^{2} f^{0}\left(t, x, z^{\delta}(t, x), \bar{u}(t, x)\right)+\delta(1-\delta) f^{0}\left(t, x, z^{\delta}(t, x), u_{2}(t, x)\right)\right. \\
& \left.+\delta(1-\delta) f^{0}\left(t, x, z^{\delta}(t, x), u_{3}(t, x)\right)+\delta^{2} f^{0}\left(t, x, z^{\delta}(t, x), u_{4}(t, x)\right)\right) \mathrm{d} t \mathrm{~d} x .
\end{aligned}
$$

\subsection{Linearized state equation}

We now would like to let $\delta \rightarrow 0^{+}$. Denote

$$
Z^{\delta}(x)=\frac{z^{\delta}(x)-\bar{z}(x)}{\delta}, \quad(t, x) \in \Omega_{T} .
$$


Then it follows from (3.10) that

$$
\left\{\begin{aligned}
\partial_{t} Z^{\delta}(t, x)- & \nabla\left(A(t, x, \bar{u}(t, x)) \nabla Z^{\delta}(t, x)\right)=\nabla \cdot\left(\frac{Q^{\delta}(t, x)-A(t, x, \bar{u}(t, x))}{\delta} \nabla z^{\delta}(t, x)\right) \\
& +(1-\delta)^{2} \int_{0}^{1} f_{z}\left(t, x, \bar{z}(t, x)+s\left(z^{\delta}(t, x)-\bar{z}(t, x)\right), \bar{u}(t, x)\right) \mathrm{d} s Z^{\delta}(t, x) \\
& +(1-\delta)\left(f\left(t, x, z^{\delta}(t, x), u_{2}(t, x)\right)-f(t, x, \bar{z}(t, x), \bar{u}(t, x))\right) \\
& +(1-\delta)\left(f\left(t, x, z^{\delta}(t, x), u_{3}(t, x)\right)-f(t, x, \bar{z}(t, x), \bar{u}(t, x))\right) \\
& +\delta\left(f\left(t, x, z^{\delta}(t, x), u_{4}(t, x)\right)-f(t, x, \bar{z}(t, x), \bar{u}(t, x))\right), \quad \text { in } \Omega_{T}, \\
Z^{\delta}(t, x)=0, & \text { on }[0, T] \times \partial \Omega, \\
Z^{\delta}(0, x)=0, & \text { in } \Omega .
\end{aligned}\right.
$$

By (S3),

$$
\left|a_{i j}(t, x, v)\right| \leq \Lambda, \quad \forall(t, x) \in \Omega_{T} ; v \in U ; 1 \leq i, j \leq n .
$$

Thus, it follows from (3.11) and Lemma 2.2 that as $\delta \rightarrow 0^{+}$,

$$
\frac{q_{i j}^{\delta}(t, x)-a_{i j}(t, x, \bar{u}(t, x))}{\delta}
$$

converges in $L^{\infty}(\Omega)$ to

$$
\begin{aligned}
& \theta_{i j}(t, x)= a_{i j}\left(t, x, u_{2}(t, x)\right)+a_{i j}\left(t, x, u_{3}(t, x)\right)-2 a_{i j}(t, x, \bar{u}(t, x)) \\
&-\frac{\left[a_{1 i}\left(t, x, u_{3}(t, x)\right)-a_{1 i}(t, x, \bar{u}(t, x))\right]\left[a_{1 j}\left(t, x, u_{3}(t, x)\right)-a_{1 j}(t, x, \bar{u}(t, x))\right]}{a_{11}\left(t, x, u_{3}(t, x)\right)}, \\
& 1 \leq i, j \leq n .
\end{aligned}
$$

On the other hand, $z^{\delta}(\cdot)$ is bounded uniformly in $L^{2}\left(0, T ; H_{0}^{1}(\Omega)\right)$. Thus, we can prove step-by-step that as $\delta \rightarrow 0^{+}, Z^{\delta}(\cdot)$ is bounded uniformly in $L^{2}\left(0, T ; H_{0}^{1}(\Omega)\right), z^{\delta}(\cdot)$ converges to $\bar{z}(\cdot)$ strongly in $L^{2}\left(0, T ; H_{0}^{1}(\Omega)\right)$, and $Z^{\delta}(\cdot)$ converges to $Z(\cdot)$ weakly in $L^{2}\left(0, T ; H_{0}^{1}(\Omega)\right)$ with $Z(\cdot)$ being the weak solution of

$$
\left\{\begin{aligned}
\partial_{t} Z(t, x)-\nabla \cdot & (A(t, x, \bar{u}(t, x)) \nabla Z(t, x))=\nabla \cdot(\Theta(t, x) \nabla \bar{z}(t, x)) \\
& +f_{z}(t, x, \bar{z}(t, x), \bar{u}(t, x)) Z(t, x)+f\left(t, x, \bar{z}(t, x), u_{2}(t, x)\right) \\
& +f\left(t, x, \bar{z}(t, x), u_{3}(t, x)\right)-2 f(t, x, \bar{z}(t, x), \bar{u}(t, x)), \quad \text { in } \Omega_{T}, \\
Z(t, x)=0, & \text { on }[0, T] \times \partial \Omega, \\
Z(0, x)=0, & \text { in } \Omega,
\end{aligned}\right.
$$

where

$$
\begin{gathered}
\Theta(t, x)=\left(\theta_{i j}(t, x)\right)=A\left(t, x, u_{2}(t, x)\right)+A\left(t, x, u_{3}(t, x)\right)-2 A(t, x, \bar{u}(t, x)) \\
-\frac{\left[A\left(t, x, u_{3}(t, x)\right)-A(t, x, \bar{u}(t, x))\right] e_{1} e_{1}^{\top}\left[A\left(t, x, u_{3}(t, x)\right)-A(t, x, \bar{u}(t, x))\right]}{e_{1}^{\top} A\left(t, x, u_{3}(t, x)\right) e_{1}} .
\end{gathered}
$$

From (3.17), we have

$$
\begin{aligned}
0 \leq & \lim _{\delta \rightarrow 0^{+}} \frac{J^{\delta}-J(\bar{u}(\cdot))}{\delta}=\int_{\Omega_{T}}\left(f_{z}^{0}(t, x, \bar{z}(t, x), \bar{u}(t, x)) Z(t, x)\right. \\
& +f^{0}\left(t, x, \bar{z}(t, x), u_{2}(t, x)\right)+f^{0}\left(t, x, \bar{z}(t, x), u_{3}(t, x)\right) \\
& \left.-2 f^{0}(t, x, \bar{z}(t, x), \bar{u}(t, x))\right) \mathrm{d} t \mathrm{~d} x .
\end{aligned}
$$




\subsection{Duality}

Let $\bar{\psi}(\cdot)$ be the solution of the adjoint equation (1.8). Then (3.23) becomes

$$
\begin{aligned}
0 \leq & \int_{\Omega_{T}}\left[f^{0}\left(t, x, \bar{z}(t, x), u_{2}(t, x)\right)+f^{0}\left(t, x, \bar{z}(t, x), u_{3}(t, x)\right)-2 f^{0}(t, x, \bar{z}(t, x), \bar{u}(t, x))\right. \\
& \left.+\left(\partial_{t} \psi(t, x)+\nabla \cdot(A(t, x, \bar{u}(t, x)) \nabla \bar{\psi}(t, x))+f_{z}(t, x, \bar{z}(t, x), \bar{u}(t, x)) \bar{\psi}(t, x)\right) Z(t, x)\right] \mathrm{d} t \mathrm{~d} x \\
= & \int_{\Omega_{T}}\left[f^{0}\left(t, x, \bar{z}(t, x), u_{2}(t, x)\right)+f^{0}\left(t, x, \bar{z}(t, x), u_{3}(t, x)\right)-2 f^{0}(t, x, \bar{z}(t, x), \bar{u}(t, x))\right. \\
& \left.+\left(-\partial_{t} Z(t, x)+\nabla \cdot(A(t, x, \bar{u}(t, x)) \nabla Z(t, x))+f_{z}(t, x, \bar{z}(t, x), \bar{u}(t, x)) Z(t, x)\right) \bar{\psi}(t, x)\right] \mathrm{d} t \mathrm{~d} x \\
= & \int_{\Omega_{T}}\left[f^{0}\left(t, x, \bar{z}(t, x), u_{2}(t, x)\right)+f^{0}\left(t, x, \bar{z}(t, x), u_{3}(t, x)\right)-2 f^{0}(t, x, \bar{z}(t, x), \bar{u}(t, x))\right. \\
& -\left(f\left(t, x, \bar{z}(t, x), u_{2}(t, x)\right)+f\left(t, x, \bar{z}(t, x), u_{3}(t, x)\right)-2 f(t, x, \bar{z}(t, x), \bar{u}(t, x))\right) \bar{\psi}(t, x) \\
& +\langle\Theta(t, x) \nabla \bar{z}(t, x), \nabla \bar{\psi}(t, x)\rangle] \mathrm{d} t \mathrm{~d} x \\
= & \int_{\Omega_{T}}\left[2\left(f(t, x, \bar{z}(t, x), \bar{u}(t, x)) \bar{\psi}(x)-f^{0}(t, x, \bar{z}(t, x), \bar{u}(t, x))-\langle A(t, x, \bar{u}(t, x)) \nabla \bar{z}(t, x), \nabla \bar{\psi}(t, x)\rangle\right)\right. \\
& -\left(f\left(t, x, \bar{z}(t, x), u_{2}(t, x)\right) \bar{\psi}(x)-f^{0}\left(t, x, \bar{z}(t, x), u_{2}(t, x)\right)-\left\langle A\left(t, x, u_{2}(t, x)\right) \nabla \bar{z}(t, x), \nabla \bar{\psi}(t, x)\right\rangle\right) \\
& -\left(f\left(t, x, \bar{z}(t, x), u_{3}(t, x)\right) \bar{\psi}(x)-f^{0}\left(t, x, \bar{z}(t, x), u_{3}(t, x)\right)-\left\langle A\left(t, x, u_{3}(t, x)\right) \nabla \bar{z}(t, x), \nabla \bar{\psi}(t, x)\right\rangle\right) \\
& -\frac{\left.\nabla \bar{z}(t, x)^{\top}\left(A\left(t, x, u_{3}(t, x)\right)-A(t, x, \bar{u}(t, x))\right) e_{1} e_{1}^{\top}\left(A\left(t, x, u_{3}(t, x)\right)-A(t, x, \bar{u}(t, x))\right) \nabla \bar{\psi}(t, x)\right] \mathrm{d} t \mathrm{~d} x}{e_{1}^{\top} A\left(t, x, u_{3}(t, x)\right) e_{1}} \\
= & \int_{\Omega_{T}}\left[2 H(t, x, \bar{z}(t, x), \bar{\psi}(t, x), \nabla \bar{z}(t, x), \nabla \bar{\psi}(t, x), \bar{u}(t, x))-H\left(t, x, \bar{z}(t, x), \bar{\psi}(t, x), \nabla \bar{z}(t, x), \nabla \bar{\psi}(t, x), u_{2}(t, x)\right)\right. \\
& -H\left(t, x, \bar{z}(t, x), \bar{\psi}(t, x), \nabla \bar{z}(t, x), \nabla \bar{\psi}(t, x), u_{3}(t, x)\right)-\Phi\left(A\left(t, x, u_{3}(t, x)\right)\right. \\
& \left.\left.-A(t, x, \bar{u}(t, x)), A\left(t, x, u_{3}(t, x)\right), \nabla \bar{z}(t, x), \nabla \bar{\psi}(t, x), e_{1}\right)\right] \mathrm{d} t \mathrm{~d} x,
\end{aligned}
$$

where $H$ is defined by (1.10),

$$
\Phi(A, B, \xi, \eta, \nu)=\frac{\eta^{\top} A \nu \nu^{\top} A \xi}{\nu^{\top} B \nu}, \quad(A, B, \xi, \eta, \nu) \in \mathcal{S}^{n} \times \mathcal{S}_{+}^{n} \times \mathbb{R}^{n} \times \mathbb{R}^{n} \times \mathbb{R}^{n}
$$

and $\mathcal{S}^{n}$ is the set of all $n \times n$ real symmetric matrices.

\subsection{Maximum condition}

By a standard argument [10], it follows from (3.24) that

$$
\begin{aligned}
& 2 H(t, x, \bar{z}(t, x), \bar{\psi}(t, x), \nabla \bar{z}(t, x), \nabla \bar{\psi}(t, x), \bar{u}(t, x)) \geq H(t, x, \bar{z}(t, x), \bar{\psi}(t, x), \nabla \bar{z}(t, x), \nabla \bar{\psi}(t, x), w) \\
& +H(t, x, \bar{z}(t, x), \bar{\psi}(t, x), \nabla \bar{z}(t, x), \nabla \bar{\psi}(t, x), v)+\Phi\left(A(t, x, v)-A(t, x, \bar{u}(t, x)), A(t, x, v), \nabla \bar{z}(t, x), \nabla \bar{\psi}(t, x), e_{1}\right), \\
& \forall v, w \in U, \quad \text { a.e. }(t, x) \in \Omega_{T} .
\end{aligned}
$$


Further, it is easy to see that (3.26) is equivalent to the following two inequalities:

$$
\begin{array}{r}
H(t, x, \bar{z}(t, x), \bar{\psi}(t, x), \nabla \bar{z}(t, x), \nabla \bar{\psi}(t, x), \bar{u}(t, x)) \geq H(t, x, \bar{z}(t, x), \bar{\psi}(t, x), \nabla \bar{z}(t, x), \nabla \bar{\psi}(t, x), v), \\
\forall v \in U, \quad \text { a.e. }(t, x) \in \Omega_{T}
\end{array}
$$

and

$$
\begin{array}{r}
H(t, x, \bar{z}(t, x), \bar{\psi}(t, x), \nabla \bar{z}(t, x), \nabla \bar{\psi}(t, x), \bar{u}(t, x)) \geq H(t, x, \bar{z}(t, x), \bar{\psi}(t, x), \nabla \bar{z}(t, x), \nabla \bar{\psi}(t, x), v) \\
+\Phi\left(A(t, x, v)-A(t, x, \bar{u}(t, x)), A(t, x, v), \nabla \bar{z}(t, x), \nabla \bar{\psi}(t, x), e_{1}\right), \\
\forall v \in U, \quad \text { a.e. }(t, x) \in \Omega_{T} .
\end{array}
$$

Moreover, we can generalize (3.28) to the following:

$$
\begin{aligned}
& H(t, x, \bar{z}(t, x), \bar{\psi}(t, x), \nabla \bar{z}(t, x), \nabla \bar{\psi}(t, x), \bar{u}(t, x)) \geq H(t, x, \bar{z}(t, x), \bar{\psi}(t, x), \nabla \bar{z}(t, x), \nabla \bar{\psi}(t, x), v) \\
&+\Phi(A(t, x, v)-A(t, x, \bar{u}(t, x)), A(t, x, v), \nabla \bar{z}(t, x), \nabla \bar{\psi}(t, x), e), \\
& \forall v \in U, e \in S^{n-1}, \quad \text { a.e. }(t, x) \in \Omega_{T},
\end{aligned}
$$

where $S^{n-1}=\left\{x \in \mathbb{R}^{n}|| x \mid=1\right\}$. Now, for given $(t, x, v)$, we denote

$$
\left\{\begin{array}{l}
\mu=\frac{A(t, x, v)^{\frac{1}{2}} e}{\left|A(t, x, v)^{\frac{1}{2}} e\right|}, \\
\xi=A(t, x, v)^{-\frac{1}{2}}[A(t, x, v)-A(t, x, \bar{u}(t, x))] \nabla \bar{z}(t, x), \\
\eta=A(t, x, v)^{-\frac{1}{2}}[A(t, x, v)-A(t, x, \bar{u}(t, x))] \nabla \bar{\psi}(t, x) .
\end{array}\right.
$$

When $e$ runs over $S^{n-1}, \mu$ will run over $S^{n-1}$. Then (3.27) and (3.28) become

$$
\begin{aligned}
H(t, x, \bar{z}(t, x), \bar{\psi}(t, x), \nabla \bar{z}(t, x), \nabla \bar{\psi}(t, x), \bar{u}(t, x)) & -H(t, x, \bar{z}(t, x), \bar{\psi}(t, x), \nabla \bar{z}(t, x), \nabla \bar{\psi}(t, x), v) \\
& \geq \sup _{|\mu|=1} \max \left(\xi^{\top} \mu \mu^{\top} \eta, 0\right) .
\end{aligned}
$$

By Lemma 2.5, one can get that

$$
\sup _{|\mu|=1} \xi^{\top} \mu \mu^{\top} \eta= \begin{cases}\frac{|\xi||\eta|+\xi^{\top} \eta}{2} & \text { if } n \geq 2 \\ \xi \eta & \text { if } n=1 .\end{cases}
$$

Thus

$$
\sup _{|\mu|=1} \max \left(\xi^{\top} \mu \mu^{\top} \eta, 0\right)=\frac{|\xi||\eta|+\xi^{\top} \eta}{2} .
$$

Combining the above, we obtain (1.9). This completes the proof of Theorem 1.1.

We can see that the limit equation (3.10) of homogenizing spike variation equation (3.2) may be different for different $r$. More precisely, (3.10) has essentially three different cases corresponding to $r<2, r=2$ and $r>2$, respectively. However, the variational equation (3.18) is independent of $r>0$. Thus, the final result (Thm. 1.1) can be got by choosing $r \in(0,2)$. Such a choice will lead to a simple proof of Theorem 1.1. But, if we did that, we would not know whether we can get other conditions from cases of $r \geq 2$. This is not satisfied.

Concerning the method to construct spike variation, we have mentioned that special forms of spike variation are needed to get good expressions of the limit equations as (3.10). When we introduce (3.1), it is natural 
to expect that $u_{4}(\cdot)$ has no essential effect on the final result. The effect of $u_{2}(\cdot)$ is in time scale. One can see that essentially, $u_{2}(\cdot)$ works as a spike variation as $A(\cdot)$ being independent of $u$. Difficulties caused by $A(\cdot)$ containing $u$ appear when $u_{3}(\cdot)$ is introduced.

Remark 3.1. If we follow the idea of sequential laminates (see Tartar [16]), we can generalize (3.10) and (3.17) by constructing more general homogenized equations with their leading terms satisfying

$$
\begin{aligned}
Q^{\delta}(t, x)= & \bar{A}(t, x)+\delta\left(A_{2}(t, x)+A_{3}(t, x)-2 \bar{A}(t, x)\right)-\delta\left(A_{3}(t, x)-\bar{A}(t, x)\right)\left(A_{3}(t, x)\right)^{-\frac{1}{2}} \\
& \times P\left(A_{3}(t, x)\right)^{-\frac{1}{2}}\left(A_{3}(t, x)-\bar{A}(t, x)\right)+o(\delta),
\end{aligned}
$$

where

$$
\bar{A}(t, x)=A(t, x, \bar{u}(t, x)), \quad A_{2}(t, x)=A\left(t, x, u_{2}(t, x)\right), \quad A_{3}(t, x)=A\left(t, x, u_{3}(t, x)\right)
$$

and

$$
\begin{aligned}
P \in \mathscr{S}_{1} & \triangleq\left\{W \in \mathcal{S}^{n} \mid W \geq 0, \operatorname{tr} W=1\right\} \\
& =\left\{\sum_{k=1}^{n} \alpha_{k} \xi_{k} \xi_{k}^{\top} \mid \alpha_{1}, \alpha_{2}, \ldots, \alpha_{n} \in[0,1], \xi_{1}, \xi_{2}, \ldots, \xi_{n} \in S^{n-1}, \sum_{k=1}^{n} \alpha_{k}=1\right\} .
\end{aligned}
$$

Consequently, we can generalize (3.21)-(3.23) with (3.22) being replaced by

$$
\begin{aligned}
\Theta(t, x)= & A_{2}(t, x)+A_{3}(t, x)-2 \bar{A}(t, x)-\left(A_{3}(t, x)-\bar{A}(t, x)\right) \\
& \times\left(A_{3}(t, x)\right)^{-\frac{1}{2}} P\left(A_{3}(t, x)\right)^{-\frac{1}{2}}\left(A_{3}(t, x)-\bar{A}(t, x)\right) .
\end{aligned}
$$

It follows from

$$
\sup _{P \in \mathscr{S}_{1}} \xi^{\top} P \eta=\sup _{|x|=1} \xi^{\top} x x^{\top} \eta
$$

that (3.21), (3.23) and (3.32) still lead to Theorem 1.1.

\section{Problem with state constraints}

In this section, we will consider the cases of state constraint. We will only state the results since the proofs are completely similar to those of elliptic cases.

(S6) Let $\mathcal{Z}$ be a Banach space with strictly convex dual $\mathcal{Z}^{*}, F: L^{2}\left(0, T ; H_{0}^{1}(\Omega)\right) \rightarrow \mathcal{Z}$ be continuous Fréchet differentiable, and $E \subseteq \mathcal{Z}$ be closed and convex.

As in Chapter 5 of [10], many state constraints can be stated in the following type:

$$
F(z(\cdot)) \in E \text {. }
$$

Let $\mathcal{P}_{\text {ad }}$ be the set of all pairs $(z(\cdot), u(\cdot))$ satisfying (1.1) and (4.1). Any $(z(\cdot), u(\cdot)) \in \mathcal{P}_{\text {ad }}$ is called an admissible pair. The set $\mathcal{U}_{\mathrm{ad}} \equiv\left\{u(\cdot) \in \mathcal{U} \mid(z(\cdot ; u(\cdot)), u(\cdot)) \in \mathcal{P}_{\mathrm{ad}}\right\}$ is called the set of admissible controls. Our optimal control problem with state constraint is:

Problem (SC). Find a control $\bar{u}(\cdot) \in \mathcal{U}_{\text {ad }}$ such that

$$
J(\bar{u}(\cdot))=\inf _{u(\cdot) \in \mathcal{U}} J(u(\cdot)) .
$$

To state necessary conditions for optimal controls of Problem (SC), we need to recall the notion of finite co-dimensionality (see Chap. 4 of [10], for example). 
Definition 4.1. Let $X$ be a Banach space and $X_{0}$ be a subspace of $X$. We say that $X_{0}$ is finite co-dimensional in $X$ if there exist $x_{1}, x_{2}, \ldots, x_{n} \in X$, such that

$$
\operatorname{span}\left\{X_{0}, x_{1}, \ldots, x_{n}\right\} \equiv \text { the space spanned by }\left\{X_{0}, x_{1}, \ldots, x_{n}\right\}=X \text {. }
$$

A subset $S$ of $X$ is said to be finite co-dimensional in $X$ if for some $x_{0} \in S, \operatorname{span}\left(S-\left\{x_{0}\right\}\right) \equiv$ the closed subspace spanned by $\left\{x-x_{0} \mid x \in S\right\}$ is a finite co-dimensional subspace of $X$ and $\overline{c o} S \equiv$ the closed convex hull of $S-\left\{x_{0}\right\}$ has a nonempty interior in this subspace.

Let $(\bar{z}(\cdot), \bar{u}(\cdot))$ be an optimal pair of Problem (SC). Let $Z=Z(\cdot ; u(\cdot)) \in L^{2}\left(0, T ; H_{0}^{1}(\Omega)\right)$ be the unique weak solution of the variational equation (3.21) and define the reachable set of variational system (3.21) as

$$
\mathcal{R}=\left\{Z(\cdot ; u(\cdot)) \mid u_{2}(\cdot), u_{3}(\cdot) \in \mathcal{U}\right\}
$$

Now, let us state the necessary conditions of an optimal control to Problem (SC) as follows:

Theorem 4.2. Let $(\mathrm{S} 1)-(\mathrm{S} 6)$ hold. Let $(\bar{z}(\cdot), \bar{u}(\cdot)) \in \mathcal{P}_{\mathrm{ad}}$ be an optimal pair of Problem (SC). Let

$$
F^{\prime}(\bar{z}(\cdot)) \mathcal{R}-E \equiv\left\{\xi-\eta \mid \xi \in F^{\prime}(\bar{z}(\cdot)) \mathcal{R}, \eta \in E\right\}
$$

be finite co-dimensional in $\mathcal{Z}$. Then there exists a triple $\left(\bar{\psi}_{0}, \bar{\psi}(\cdot), \bar{\varphi}(\cdot)\right) \in \mathbb{R} \times L^{2}\left(0, T ; H_{0}^{1}(\Omega)\right) \times \mathcal{Z}^{*}$ satisfying

$$
\begin{aligned}
& \left\{\begin{array}{l}
\bar{\psi}_{0} \leq 0, \\
\left(\bar{\psi}_{0}, \bar{\varphi}(\cdot)\right) \neq 0, \\
\left(\bar{\psi}_{0}, \bar{\psi}(\cdot)\right) \neq 0, \quad \text { if } F^{\prime}(\bar{z}(\cdot))^{*} \text { is injective },
\end{array}\right. \\
& \langle\bar{\varphi}(\cdot), \eta-F(\bar{z}(\cdot))\rangle \mathcal{Z}^{*}, \mathcal{Z} \leq 0, \quad \forall \eta \in E, \\
& \left\{\begin{aligned}
\partial_{t} \bar{\psi}(t, x)+\nabla \cdot(A(t, x, \bar{u}(t, x)) \nabla \bar{\psi}(t, x))=-\psi_{0} f_{z}^{0}(t, x, \bar{z}(t, x), \bar{u}(t, x)) \\
\quad-f_{z}(t, x, \bar{z}(t, x), \bar{u}(t, x)) \bar{\psi}(t, x)+F^{\prime}(\bar{z}(\cdot))^{*} \bar{\varphi}, \quad \text { in } \Omega_{T}, \\
\bar{\psi}(t, x)=0, \quad \text { on }[0, T] \times \partial \Omega, \\
\bar{\psi}(T, x)=0, \quad \text { in } \Omega,
\end{aligned}\right. \\
& H(t, x, \bar{z}(t, x), \bar{\psi}(t, x), \nabla \bar{z}(t, x), \nabla \bar{\psi}(t, x), \bar{u}(t, x))-H(t, x, \bar{z}(t, x), \bar{\psi}(t, x), \nabla \bar{z}(t, x), \nabla \bar{\psi}(t, x), v) \\
& \geq \frac{1}{2}\left|A(t, x, v)^{-\frac{1}{2}}(A(t, x, \bar{u}(t, x))-A(t, x, v)) \nabla \bar{z}(t, x)\right|\left|A(t, x, v)^{-\frac{1}{2}}(A(t, x, \bar{u}(t, x))-A(t, x, v)) \nabla \bar{\psi}(t, x)\right| \\
& +\frac{1}{2}\left\langle A(t, x, v)^{-\frac{1}{2}}(A(t, x, \bar{u}(t, x))-A(t, x, v)) \nabla \bar{z}(t, x), A(t, x, v)^{-\frac{1}{2}}(A(t, x, \bar{u}(t, x))-A(t, x, v)) \nabla \bar{\psi}(t, x)\right\rangle, \\
& \forall v \in U, \quad \text { a.e. }(t, x) \in \Omega_{T},
\end{aligned}
$$

where

$$
\begin{array}{r}
H(t, x, z, \psi, \xi, \eta, v)=\langle\psi, f(t, x, z, v)\rangle+\psi_{0} f^{0}(t, x, z, v)-\langle A(t, x, v) \xi, \eta\rangle \\
(t, x, z, \psi, \xi, \eta, v) \in[0, T] \times \Omega \times \mathbb{R} \times \mathbb{R} \times \mathbb{R}^{n} \times \mathbb{R}^{n} \times U
\end{array}
$$

Acknowledgements. The author thanks the anonymous referees for their helpful suggestions.

\section{REFERENCES}

[1] G. Allaire, Homogenization and two-scale convergence. SIAM J. Math. Anal. 23 (1992) 1482-1518.

[2] A. Bensoussan, J.L. Lions and G. Papanicolaou, Asymptotic Analysis for Periodic Structures. North-Holland Company, Amsterdam (1978). 
[3] C. Calvo-Jurado and J. Casado-Diaz, Homogenization of Dirichlet parabolic problems for coefficients and open sets simultaneously variable and applications to optimal design. J. Comput. Appl. Math. 192 (2006) 20-29.

[4] J. Casado-Diaz, J. Couce-Calvo and J.D. Martin-Gómez, Optimality conditions for nonconvex multistate control problems in the coefficients. SIAM J. Control Optim. 43 (2004) 216-239.

[5] E. Casas, Optimal Control in coefficients of elliptic equations with state constraints. Appl. Math. Optim. 26 (1992) $21-37$.

[6] I. Ciuperca, M. El Alaoui Talibi and M. Jai, On the optimal control of coefficients in elliptic problems, Application to the optimization of the head slider. ESAIM: COCV 11 (2005) 102-121.

[7] H. Gao and X. Li, Necessary conditions for optimal control of elliptic systems. J. Australian Math. Soc. Ser. B 41 (2000) $542-567$.

[8] A. Holmbom, Homogenization of parabolic equations an alternative approach and some corrector-type results. Appl. Math. 42 (1997) 321-343.

[9] O.A. Ladyženskaja, V.A. Solonnikov and N.N. Ural'ceva, Linear and Quasi-linear Equations of Parabolic Type, Transl. Math. Monographs 23. American Mathematical Society, Providence (1968).

[10] X. Li, and J. Yong, Optimal Control Theory for Infinite Dimensional Systems. Birkhäuser, Boston (1995).

[11] H. Lou and J. Yong, Optimality Conditions for Semilinear Elliptic Equations with Leading Term Containing Controls. SIAM J. Control Optim. 48 (2009) 2366-2387.

[12] F. Murat and L. Tartar, Calculus of variations and homogenization, in Topics in the Mathematical Modelling of Composite Materials, Progress in Nonlinear Diffrential Equations and their Applications 31, L. Cherkaev and R.V. Kohn Eds., Birkaüser, Boston (1998) 139-174.

[13] U. Raitums and W.H. Schmidt, On necessary optimal conditions for optimal control problems governed by elliptic systems. Optimization 54 (2005) 149-160.

[14] S.Y. Serovajsky, Sequential extension in the problem of control in coefficients for elliptic-type equations. J. Inverse Ill-Posed Probl. 11 (2003) 523-536.

[15] R.K. Tagiyev, Optimal control by the coefficients of a parabolic equation. Trans. Acad. Sci. Azerb. Ser. Phys.-Tech. Math. Sci. Math. Mech. 24 (2004) 247-256.

[16] L. Tartar, Estimations fines de coefficients homogénéisés, Ennio de Giorgi Colloquium, in Pitman Research Notes in Mathematics 125, P. Krée Ed., Pitman, London (1985) 168-187. 